\title{
Lentivirus-mediated microRNA-124 gene-modified bone marrow mesenchymal stem cell transplantation promotes the repair of spinal cord injury in rats
}

\author{
Jia-Lin Song ${ }^{1,3}$, Wei Zheng ${ }^{1,3}$, Wei Chen ${ }^{1}$, Yun Qian ${ }^{1}$, Yuan-Ming Ouyang ${ }^{2}$ and Cun-Yi Fan ${ }^{1}$
}

Our study aims to explore the effects of lentivirus-mediated microRNA-124 (miR-124) gene-modified bone marrow mesenchymal stem cell (BMSC) transplantation on the repair of spinal cord injury $(\mathrm{SCI})$ in rats. BMSCs were isolated from the bone marrow of rats. The target gene miR-124 was identified using a luciferase-reporter gene assay. Seventy-two rats were selected for construction of the $\mathrm{SCl}$ model, and the rats were randomly divided into the blank group, sham group, $\mathrm{SCl}$ group, negative control (NC) group, overexpressed miR-124 group and si-PDXK group. The mRNA expression of miR-124 and the mRNA and protein expression of pyridoxal kinase (PDXK) were detected by quantitative real-time polymerase chain reaction and western blotting. The locomotor capacity of the rats was evaluated using the Basso, Beattie and Bresnahan (BBB) scale. Brdu, neuron-specific enolase (NSE), neurofilament (NF) and microtubule-associated protein 2 (MAP2) were detected using immunohistochemistry. The expression levels of thyrotropin-releasing hormone (TRH), prostacyclin (PGI2) and gangliosides (GM) were measured using an enzyme-linked immunosorbent assay. PDXK was identified as the target gene of miR-124. The overexpressed miR-124 group exhibited higher miR-124 expression than the SCI, NC and si-PDXK groups. Compared with the SCI and NC groups, the PDXK expression was downregulated in the overexpressed miR-124 and si-PDXK groups, and the BBB scores were significantly increased 7, 21 and 35 days after transplantation. The double-labeled positive cell densities (Brdu+NSE/NF/MAP2) and the expression levels of TRH, PGI2 and GM in the overexpressed miR-124 group were significantly higher than those in the NC and SCI groups. These results indicated that miR-124 targeted PDXK to accelerate the differentiation of BMSCs into neurocytes and promote $\mathrm{SCl}$ repair.

Experimental \& Molecular Medicine (2017) 49, e332; doi:10.1038/emm.2017.48; published online 19 May 2017

\section{INTRODUCTION}

The spinal cord is the main channel for motor and sensory conduction between the brain and body. ${ }^{1}$ Spinal cord injury (SCI), prevalent in young patients, is one of the most common diseases worldwide with a high incidence, a high disability rate and a high cost for treatment. ${ }^{2}$ SCI is characterized by neuron and oligodendrocyte loss, demyelination, axonal injury and axonal myelination disorder, resulting in sensation loss associated with hemiplegia or paralysis. ${ }^{3}$ Currently, vehicle crashes are the leading cause of SCI, followed by falls, acts of violence (mainly gunshot wounds) and sports/recreational activities. ${ }^{4}$ Conventional therapy, cell transplantation and gene therapy are the main therapies used for treating SCI. For cell transplantation, bone marrow mesenchymal stem cells (BMSCs) are transplantable cells that are easily obtained and have no immune rejection reaction. Gene therapy can be used to import neurotrophic factors into the body and promote SCI recovery. ${ }^{5,6}$ The regulation of microRNAs (miRs) in gene therapy has attracted more and more attention in recent years, and it may provide a new target for SCI treatment. ${ }^{7}$

As non-coding small-molecule RNAs, miRs can regulate gene expression. ${ }^{8}$ miR-124 is widely expressed in the mature mammalian brain and is also one of the earliest discovered highly conserved miRs; it plays an important role in the process of neurogenesis. ${ }^{9,10} \mathrm{Xu}$ et al. ${ }^{11}$ reported that the expression of miR-124 is associated with BMSCs and spinal cord stem cells, which have an important influence on functional restoration after SCI. Mesenchymal stem cells (MSCs), characterized by multi-potent differentiation, are a stem cell type found in the bone marrow $(\mathrm{BM})$ and other tissues. ${ }^{12}$ BMSCs are a type of

\footnotetext{
${ }^{1}$ Department of Orthopedics, Shanghai Jiao Tong University Affiliated Sixth People's Hospital, Shanghai, China and ${ }^{2}$ Department of Orthopedics, The East Campus of Shanghai Sixth People's Hospital, Shanghai University of Medical and Health, Shanghai, China

${ }^{3}$ These authors contributed equally to this work.

Correspondence: Dr C-Yi Fan, Department of Orthopedics, Shanghai Jiao Tong University Affiliated Sixth People's Hospital, No. 600, Yishan Road, Xuhui District, Shanghai 200233, China.

E-mail: cyfan9876@163.com

Received 1 September 2016; revised 2 December 2016; accepted 9 December 2016
} 
hematopoietic stem cell in the BM that has a high degree of self-renewal and differentiation potential. ${ }^{13}$ There are many advantages in the application of stem cells, especially for BMSCs, which simultaneously repair cartilage and bone with positive effects and contribute to the reconstruction and integration of the host surface. ${ }^{14}$ BMSC transplantation provides a new direction for SCI treatment, which was first performed in a rat model of SCI. ${ }^{15}$ As there were relatively scarce reports on the mechanism of miR-124 gene-modified BMSC transplantation for the repair of SCI, this study aims to explore the effects of lentivirus-mediated miR-124 genemodified BMSC transplantation on the repair of SCI in rats in order to provide targets for the treatment of SCI.

\section{MATERIALS AND METHODS}

\section{Ethic statement}

This study was performed in accordance with the animal ethics standards and with approval of the animal ethics committee at Shanghai Jiao Tong University Affiliated Sixth People's Hospital. All the procedures used in our animal experiments were in accordance with the standard of experimental animal management and protection principles.

\section{Isolation and identification of BMSCs}

Rats were anesthetized with an intra-peritoneal injection of ketamine $\left(60 \mathrm{mg} \mathrm{kg}^{-1}\right)$ at first. Their four limbs were removed after iodine disinfection. The bone marrow cavity was exposed with an incision in the metaphysis and rinsed with phosphate-buffered saline (PBS) until the fluid was clear. The bone marrow cell suspension was prepared, followed by centrifugation at 1500 r.p.m. for $5 \mathrm{~min}$. It was then re-suspended in Dulbecco's modified Eagle's medium (DMEM) containing $10 \%$ fetal bovine serum (FBS), $100 \mathrm{U} \mathrm{ml}^{-1}$ of penicillin and $100 \mathrm{mg} \mathrm{ml}^{-1}$ of streptomycin. The cells were cultured in DMEM at $37^{\circ} \mathrm{C}, 5 \% \mathrm{CO}_{2}$ and saturated humidity. The medium was changed for the first time after 3 days, and non-adherent cells were discarded. Thereafter, the medium was changed every 2 days until cells reached a high confluence. Then, the cells were passaged and cryopreserved after trypsin digestion. The cells were rinsed twice with PBS, and $0.25 \%$ trypsin was added to adjust the density to $10^{5} \mathrm{ml}^{-1}$. Then, after the addition of fluorescent-labeled antibodies (anti-rat CD29/CD34/ CD44/CD45 antibodies from Abcam, Cambridge, MA, USA), the cells were incubated in the dark for $30 \mathrm{~min}$ at $4{ }^{\circ} \mathrm{C}$. After centrifugation at $200 \mathrm{~g}$, the cell debris were re-suspended in PBS, and the surface markers for BMSCs (cells positive for CD29, CD34, CD44 and CD45) were identified using a flow cytometer (Beckton Dickinson, BD Bioscience, San Jose, CA, USA).

\section{Construction and identification of the recombinant lentiviral vector}

Polymerase chain reaction (PCR) amplification was applied to obtain DNA fragments containing the miR-124 sequences in rats (100 bp upstream and downstream sequences). The DNA fragments obtained from PCR were inserted into the H1-MCS-CMV-EGFP vector and sequenced using dual-enzyme digestion with $\mathrm{XhoI}$ and BamHI. The PCR conditions were $5 \mathrm{~min}$ of pre-denaturation at $95^{\circ} \mathrm{C} ; 25$ reaction cycles of $30 \mathrm{~s}$ denaturation at $95^{\circ} \mathrm{C}, 30 \mathrm{~s}$ annealing at $58^{\circ} \mathrm{C}$ and $30 \mathrm{~s}$ extension at $72{ }^{\circ} \mathrm{C}$; followed by $5 \mathrm{~min}$ of final extension at $72^{\circ} \mathrm{C}$. The PCR primer sequences were as follows: forward: $5^{\prime}$-TCGTTAAGGCA CGCGGTG-3'; reverse: 5'-GTGCAGGGTCCGAGGT-3'. The 293T cells were co-transfected with the recombinant lentiviral vector and two auxiliary packaging plasmids (pHelper1.0 and pHelper2.0), followed by cell culture for $48 \mathrm{~h}$. Then, the supernatant was collected from the cells and filtered through a $0.45-\mu \mathrm{m}$ membrane. Finally, the recombinant lentiviral vector containing miR-124 (miR-124-LV) and the recombinant lentiviral vector containing the green fluorescent protein (GFP) reporter gene (NC-EGFP-LV) were obtained. The virus titer of the cells was determined using a gradient dilution method in a 24-well plate. At first, $293 \mathrm{~T}$ cells were seeded into the 24 -well plate at a density of $1 \times 10^{5}$ cells per well. After the cells were cultured for $24 \mathrm{~h}$, the virus-containing fluid $(50 \mu \mathrm{l})$ was serially diluted at gradient proportions (ranging from $10^{-1}$ to $10^{-6}$ ) as required. Each concentration of the fluid was added to three wells for transfection. Forty-eight hours after transfection, the diluted concentration of cells that was $\sim 10 \%$ of the GFP expression frequency was selected for later experiments. The number of GFP positive cells and the virus titer were measured for cells at this concentration using the following calculation: virus titer $\left(\mathrm{BT}=\mathrm{TU} \mathrm{ml}{ }^{-1}\right) \mathrm{TU} \mu \mathrm{l}^{-1}=(P \times N / 100 \times V) \times 1 / \mathrm{DF}$ ( $P=$ GFP positive cells, $N=10^{5}, V=$ virus fluid volume $=50 \mu \mathrm{l}$, $\mathrm{DF}=$ dilution proportion).

The recombinant lentiviral vector was transfected into BMSCs in the logarithmic phase of growth (multiplicity of infection (MOI) = 20). Three days after the transfection, 4',6-diamidino-2-phenylindole (DAPI) staining was implemented for $1 \mathrm{~h}$. Then, the expression of the lentivirus reporter gene GFP was observed under a fluorescence microscope, and the cells were sub-cultured for subsequent experiments when the transfection efficiency $>95 \%$.

\section{Construction and identification of the PDXK-siRNA (si-PDXK) plasmid}

On the basis of the published pyridoxal kinase (PDXK) mRNA sequence (GenBank: NM 031769.1) and siRNA design principles, the DNA template for the target gene was synthesized with the online software provided by the Promega Corporation (Madison, WI, USA), followed by in vitro transcription. Thus, the final si-PDXK plasmid was obtained. Once the siRNA efficiency in BMSCs was observed to be higher than $85 \%$ with an effective duration time of 4-10 days, the si-PDXK plasmid was identified as viable and was reserved for later experiments. The sequence of the positive-sense strand was $5^{\prime}$-GCA AACAAUGUCAACAAGU dTdT- $3^{\prime}$ and that of the antisense strand $3^{\prime}$-dTdT CGUUUGUUACAGUUGUUCA- ${ }^{\prime}$. Then, the BMSCs were transfected with the si-PDXK plasmid using Lipofectamine 2000 (Lip2000; Invitrogen, Carlsbad, CA, USA).

\section{Luciferase-reporter gene assay}

The target gene of miR-124 was predicted by the online software Targetscan 7.1 (http://www.targetscan.org/vert_71/). DNA fragments containing the miR-124-binding site in the $3^{\prime}$-untranslated regions ( $3^{\prime}$-UTR) of PDXK and fragments containing the mutant miR-27abinding site were separately inserted into the luciferase reporter gene plasmid to obtain the PDXK-WT (wild type) and PDXK-MUT (mutant type) plasmids, respectively. Afterwards, the miR-124 mimics and PDXK-WT/PDXK-MUT plasmids were co-transfected into 293T cells, and the luciferase activities of the transfected cells were detected using luciferase assay. In addition, 293T cells were co-transfected with a negative control (NC) oligonucleotide and PDXK-WT/PDXK-MUT plasmids as control. 


\section{A rat model of SCI}

Seventy-two specific pathogen-free (SPF) Sprague-Dawley (SD) rats between 2 and 3 months of age, and weighing 200-250 g, were purchased from Shanghai Biomodel Organism Science \& Technology Co. The improved Allen method was applied to construct the SCI model. A hitting device was placed on a stereotaxic apparatus. After the T10 spinal cord was exposed, a circular thin copper gasket (diameter $=3 \mathrm{~mm}$, area $=7 \mathrm{~mm}^{2}$, weight $=0.1 \mathrm{~g}$ ) was placed on the surface of the T10 spinal cord. A weight of $10 \mathrm{~g}$ fell free onto the gasket to cause SCI. The amount of the injury was set as $10 \mathrm{~g} .5 \mathrm{~cm}$. A sham operation (sham) group was used as the control, and T10 spinal cord was exposed in the same way without SCI. All 72 SD rats were randomly divided into six groups. In the blank group $(n=12)$, rats were administered $100 \mu \mathrm{l}$ of sterile saline using a microsyringe via the tail vein. In the sham group, rats underwent a sham operation of SCI and were administered $100 \mu$ of sterile saline using a microsyringe via the tail vein. In the SCI group, rats were administered $100 \mu \mathrm{l}$ of sterile saline using a microsyringe via the tail vein of rats 1 day after the success of the SCI model. In the negative control (NC) group, rats were administered $2 \times 10^{6}$ per $100 \mu \mathrm{l}$ of sterile saline and transplanted with BMSCs that were transfected with the NC-EGFP-LV vector through a microsyringe 1 day after the success of the SCI model. In the overexpressed miR-124 group $(n=12)$, rats were administered with $2 \times 10^{6}$ per $100 \mu \mathrm{l}$ of sterile saline and transplanted with BMSCs that were transfected with the miR-124-LV vector through a microsyringe 1 day after the success of the SCI model. In the si-PDXK group, rats were administered with $2 \times 10^{6}$ per $100 \mu \mathrm{l}$ of sterile saline and transplanted with BMSCs that were transfected with the si-PDXK plasmid.

\section{Quantitative real-time polymerase chain reaction}

Thirty-five days after the BMSCs were transplanted into rats, injured spinal cord tissue homogenate was extracted from the rats. The total RNA was extracted from the tissues using an RNAiso Plus Kit (Takara Biotechnology, Dalian, Liaoning, China). The PrimeScript RT reagent kit (Takara Biotechnology) was applied for reverse transcription. The quantitative real-time polymerase chain reaction was conducted using a SteponePlus (Applied Biosystems, Grand Island, NY, USA) PCR instrument. The PCR reaction volumes contained $1.6 \mu \mathrm{l}$ of $\mathrm{cDNA}$ solution, $5 \mu$ l of $2 \times$ SYBR Green Taq PCR mix (Takara Biotechnology), $0.2 \mu \mathrm{l}$ of PCR forward primer and $0.2 \mu \mathrm{l}$ of reverse primer (both at $10 \mu \mathrm{M})$ and $3 \mu \mathrm{l}$ of double distilled water. The PCR reaction conditions were as follows: $5 \mathrm{~min}$ pre-denaturation at $95^{\circ} \mathrm{C} ; 60$ cycles of $10 \mathrm{~s}$ denaturation at $95^{\circ} \mathrm{C}, 10 \mathrm{~s}$ annealing at $58^{\circ} \mathrm{C}$, and extension $10 \mathrm{~s}$; followed by a final $10 \mathrm{~min}$ extension at $72{ }^{\circ} \mathrm{C}$. With $\beta$-actin (Gene ID 2597) selected as the internal reference, the PDXK and miR-124 mRNA expression was detected using the $2^{-\Delta \Delta C t}$ method. The primer sequences are shown in Table 1.

\section{Table 1 Primer sequences for quantitative real-time} polymerase chain reaction

\begin{tabular}{ll}
\hline Gene & Primer sequence \\
\hline PDXK & F: 5'-AGACATTATCACGCCCAACC-3' \\
miR-124 & R: 5'-CCAGTGCCCACAAAGACG-3' \\
& F: 5'-TCGTTAAGGCACGCGGTG-3' \\
$\beta$-actin & R: 5'-GTGCAGGGTCCGAGGT-3' \\
& F: 5'-TGGGACGACATGGAGAAAA-3' \\
& R: 5'-CTGGAAGGTGGACAGCGAG-3'
\end{tabular}

Abbreviations: $F$, forward; $R$, reverse.

\section{Western blotting}

The injured spinal cord tissues were extracted 35 days after the BMSCs were transplanted into rats. The tissue homogenate was obtained using Radio-Immunoprecipitation Assay (RIPA) lysis buffer (Gibco, Grand Island, NY, USA) and protease inhibitors (Sigma-Aldrich Chemical Company, St Louis, MO, USA) that were added to tissues, followed by centrifugation ( $12000 \mathrm{~g}$ for $10 \mathrm{~min}$ ) at $4{ }^{\circ} \mathrm{C}$. The supernatant was then extracted as protein samples, and the protein concentration of these samples was detected using a bicinchoninic acid (BCA) kit (Bio-Rad Laboratories, CA, USA). After detection, the samples were preserved at $-80^{\circ} \mathrm{C}$. Western blotting was performed using $10 \%$ sodium dodecyl sulfate-polyacrylamide gel electrophoresis with the addition of $20 \mu \mathrm{g}$ of protein from each sample into each well. After the primary antibody against anti-PDXK (sc-50961, 1:200, Santa Cruz Biotechnology, CA, USA) was added, the protein samples were preserved overnight at $4{ }^{\circ} \mathrm{C}$. A peroxidase (POD)-conjugated goat anti-rabbit (1:5000) antibody was used as the secondary antibody. It was added to the samples, and this was followed by culture for $30 \mathrm{~min}$ at room temperature. Horseradish peroxidase (HRP; Bio-Rad Laboratories) was added to the samples for color development. ImageQuant 350 and ImageQuant TL-1 (GE Healthcare, Fairfield, CT, USA) were applied for band analysis of the protein samples. The protein $\beta$-actin was used as the internal reference, and the gray-scale ratio of the target protein and $\beta$-actin was represented as the relative protein expression.

\section{Basso, Beattie and Bresnahan scale}

The Basso, Beattie and Bresnahan (BBB) scale was used to assess the locomotor capacity following SCI after $24 \mathrm{~h}$ of spinal cord contusion and at 7, 21 and 35 days after the transplantation of BMSCs. Assessments of four aspects were conducted, including limb joint performance, paw placement, toe clearance and the overall behavioral performance. $^{16}$

\section{Immunohistochemistry}

The extracted spinal cord tissues were rinsed with PBS three times, fixed with $4 \%$ paraformaldehyde, embedded in paraffin and finally sliced into sections. Then, the tissue sections were stained using immunohistochemistry. After dewaxing and rehydration, 3\% hydrogen peroxide was added to the sections for $15 \mathrm{~min}$ to block the endogenous peroxidase activity, followed by antigen retrieval with $30 \mathrm{~min}$ steam-heating. After that, the tissues were sealed in PBS containing $1 \%$ bovine serum albumin for $1 \mathrm{~h}$ at room temperature, and the samples were incubated for $30 \mathrm{~min}$ at room temperature with the addition of the primary antibodies, followed by an overnight incubation at $4{ }^{\circ} \mathrm{C}$. The primary antibodies included those against neuron-specific enolase (NSE, ab53025; 1:250; Abcam), neurofilament (NF, ab7795; 1:200; Abcam) and microtubule-associated protein 2 (MAP2, ab5392; 1:250; Abcam), which were added to the tissue sections together with the antibody against bromodeoxyuridine (BrdU, ab6326; 1:250; Abcam). Then, the tissues were rinsed with PBS three times ( 3 min per time), and the POD-conjugated goat anti-rabbit (1:5000) secondary antibody was added dropwise. The sections were finally preserved at room temperature for $30 \mathrm{~min}$. Then, they were rinsed with PBS three times (3 min per time) and HRP (Bio-Rad Laboratories) was added to the sections for color development. After this, the sections were subjected to hematoxylin counter-staining, alcohol dehydration and mounting with neutral gum. The stained tissues were observed under a microscope and photographed at $\times 400$ magnification. Ten fields were randomly selected to measure the densities of the double-labeled positive cells (BrdU+NSE, BrdU+NF, 
BrdU+MAP2) and the BrdU-positive cells (cell per $\mathrm{mm}^{3}$ ). The ratio of the BrdU-positive cells/nerve cell marker-positive cells to the BrdU-positive cells/nerve cell marker-negative cells was calculated.

\section{Enzyme-linked immunosorbent assay}

Injured tissues were collected and rinsed with PBS 3 times, and the tissue homogenate was prepared with the addition of RIPA lysis buffer (Gibco, Grand Island, NY, USA) and protease inhibitors (Sigma-Aldrich Chemical Company, St. Louis, MO, USA). Then, the homogenate was centrifuged $(12000 \times g)$ for $10 \mathrm{~min}$ at $4{ }^{\circ} \mathrm{C}$, and the supernatant was extracted as protein samples. The expression of the SCI repair-related proteins was detected using ELISA kits (R\&D Systems, Minneapolis, MN, USA) containing thyrotropin-releasing hormone (TRH), prostacyclin (PGI2) and gangliosides (GM).

\section{Statistical analysis}

SPSS 20.0 software (SPSS, Chicago, IL, USA) was applied for statistical analysis, and the measurement data are represented as the mean \pm s.d. $(\bar{x} \pm \mathrm{s})$. Comparisons between two groups were analyzed using a $t$-test. A one-way analysis of variance was used for comparisons among multiple groups, and the least significant difference method was implemented with pairwise comparisons. $P<0.05$ was considered statistically significant.

\section{RESULTS}

Morphological characteristics and surface markers of BMSCs after in vitro culture

The cell morphology of BMSCs after in vitro culture and subculture (three generations) was in accordance with the morphological characteristics of BMSCs. BMSCs were observed as macromonocytes in various forms with fusiform, polygonal or star-like neurites, and the cells were present as single cells or multiple-cell clones with rapid cell proliferation after 3-7 days of primary culture. Ten days after the primary culture, fusiform BMSCs were observed and reached a high confluence (Figure 1). Flow cytometry was applied to detect the surface markers for BMSCs. The results showed that the percentages of positive CD29, CD44, CD34 and CD45 cells were 88.75\%, $75.39 \%, 1.11 \%$ and $0.71 \%$, respectively, which were all in

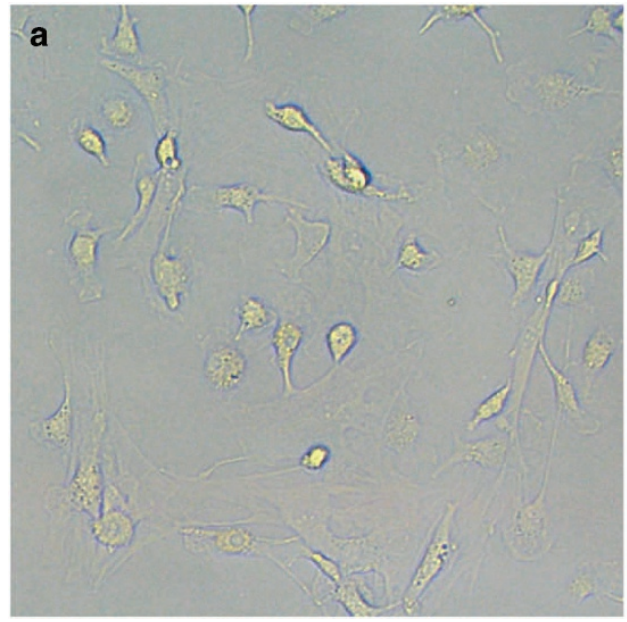

compliance with the typical BMSC characteristics (Figure 2). These results confirmed that BMSCs were successfully isolated from rats and cultured.

Transfection efficiency of the recombinant lentiviral vector GFP-positive cells were detected at $72 \mathrm{~h}$ after the BMSCs were transfected with the recombinant lentivirus vector. The percentage of GFP-positive cells was higher than 95\%, which shows that the transfection was successful (Figure 3).

\section{miR-124 targets $P D X K$, as identified by a luciferase assay} Shown as Figure 4, the Targetscan software identified that PDXK was the target gene of miR-124. miR-124 mimics had no effect on the luciferase activity of 293 T cells with PDXK-MUT. However, the luciferase activity of the cells with NC+PDXKWT was significantly higher than that of the cells with miR-124 mimics $P D X K-W T(P<0.05)$. Thus, the miR-124 mimics decrease the luciferase activity of cells with $P D X K-W T$.

\section{Comparisons of the mRNA expression of miR-124 and PDXK mRNA among the blank, sham, SCI, NC, overexpressed miR-124 and si-PDXK groups}

The mRNA expression levels of miR-124 and PDXK detected by quantitative real-time polymerase chain reaction are shown in Figure 5. The miR-124 mRNA expression levels in the SCI, $\mathrm{NC}$ and si-PDXK groups were significantly decreased in comparison to the blank and sham groups (all $P<0.05$ ), and the miR-124 mRNA expression levels in the NC and si-PDXK groups were markedly higher than that in the SCI group (both $P<0.05)$. The overexpressed miR-124 group exhibited a significantly higher miR-124 mRNA expression than the SCI and NC groups (both $P<0.05$ ), but it was not notably different compared with the blank and the sham groups (both $P>0.05$ ). PDXK mRNA expression levels in the SCI and NC groups were significantly elevated in comparison to the blank and sham groups (all $P<0.05$ ), and the SCI group had a higher PDXK mRNA expression than the NC group $(P<0.05)$. The mRNA expression of PDXK in the overexpressed miR-124 group was

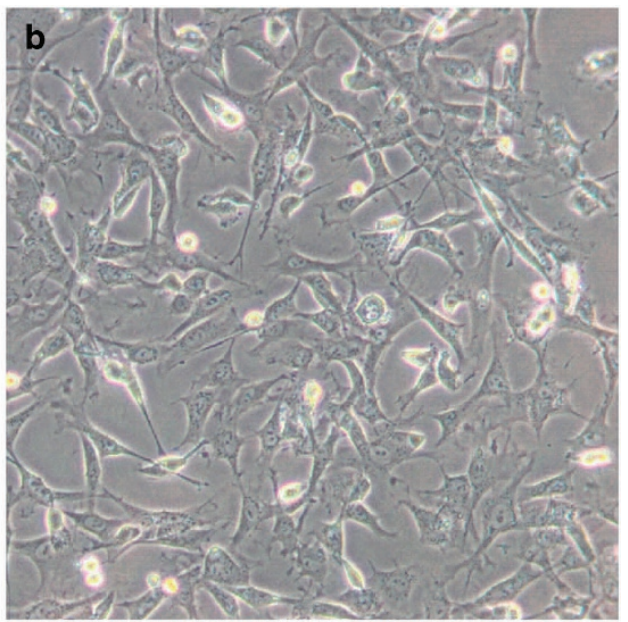

Figure 1 The cell morphology of BMSCs after in vitro culture and subculture ( $\times 200)$. (a) The cell morphology of BMSCs 3 days after the primary culture. (b) The cell morphology of BMSCs 7 days after the primary culture. BMSCs, bone marrow mesenchymal stem cells. 

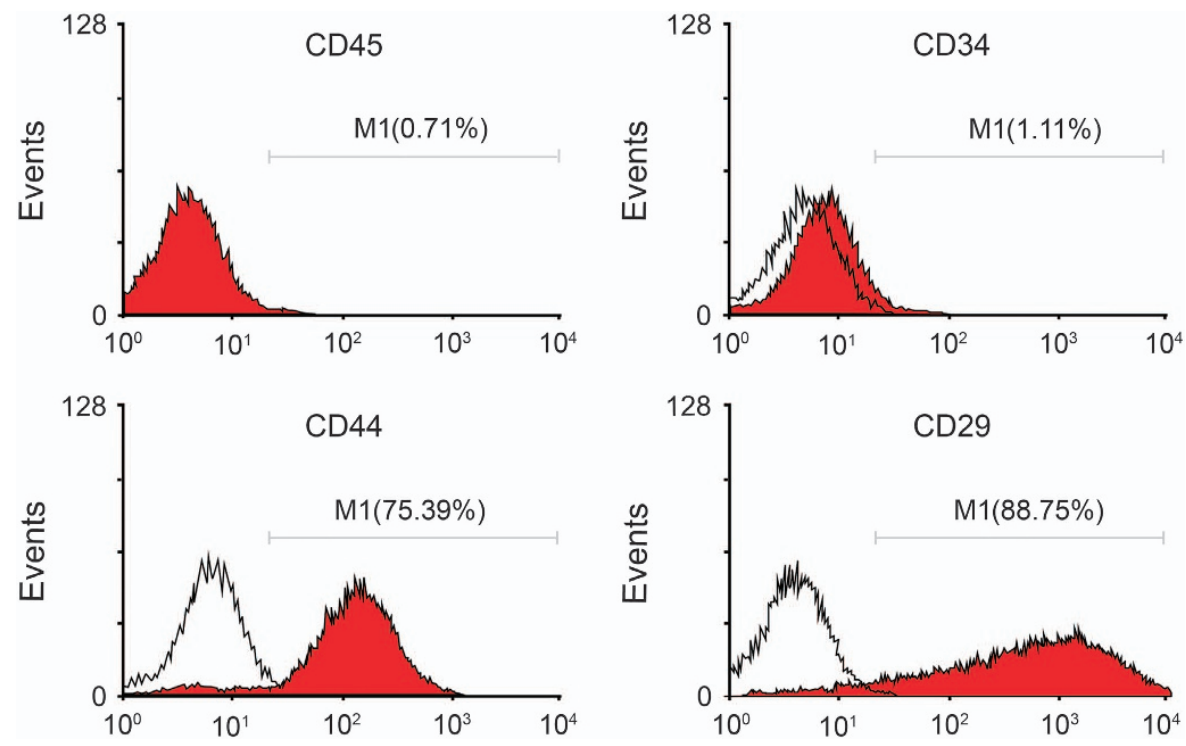

Figure 2 The positive rates of the surface markers (CD29, CD44, CD34 and CD45) of BMSCs detected by flow cytometry. BMSCs, bone marrow mesenchymal stem cells.
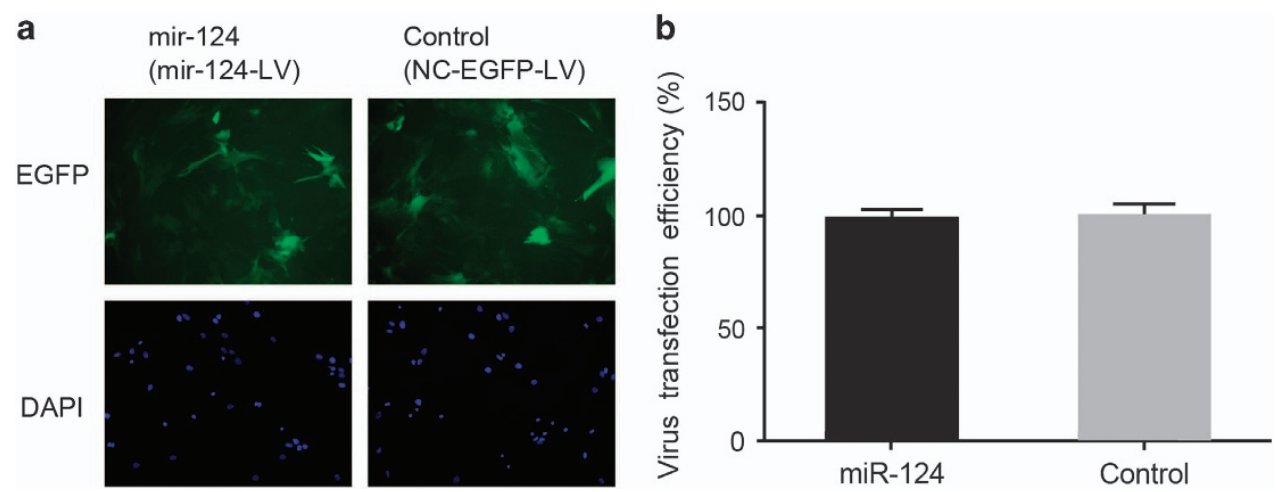

Figure 3 The transfection efficacy of recombinant lentiviral vector in BMSCs. (a) The fluorescence image of BMSCs transfected with miR-124-LV or NC-EGFP-LV. (b) A comparison of the rate of EGFP-positive cells (transfection efficacy) between BMSCs transfected with miR-124-LV or NC-EGFP-LV. BMSCs, bone marrow mesenchymal stem cells; miR-124, microRNA-124; miR-124-LV, the recombinant lentiviral vector containing the miR-124 gene; NC-EGFP-LV, the recombinant lentiviral vector containing green fluorescent protein reporter gene.

significantly lower than that of the SCI and NC groups (both $P<0.05)$. However, no significant difference was observed in the overexpressed miR-124 group compared with the blank and sham groups (both $P>0.05$ ). These results indicated that the overexpression of miR-124 inhibited the expression of PDXK.

Comparison of the PDXK protein expression among the blank, sham, SCI, NC, overexpressed miR-124 and si-PDXK groups

The protein expression of PDXK detected by western blotting is shown in Figure 6. Compared with the blank and sham groups, the PDXK protein expression levels in the SCI and NC groups were significantly increased (both $P<0.05$ ), and the SCI group showed a higher PDXK protein expression level than the NC group $(P<0.05)$. In addition, the PDXK protein expression levels in the overexpressed miR-124 and si-PDXK groups were significantly lower compared with the SCI and NC groups (both $P<0.05)$. However, the PDXK protein expression levels in the overexpressed miR-124 and si- $P D X K$ groups were similar to those of the blank and the sham groups $(P>0.05)$. These results further demonstrated that the overexpression of miR-124 inhibited the expression of PDXK.

\section{Comparison of the BBB scores at different time points} among the blank, sham, SCI, NC, overexpressed miR-124 and si-PDXK groups

The BBB scores are presented in Table 2. Twenty-four hours after being injured, the BBB scores in the SCI, NC, overexpressed miR-124 and si-PDXK groups were significantly lower than that in the blank and sham groups (all $P<0.05$ ). In addition, 7 days and 24 days after the BMSCs were transplanted into rats, the BBB scores in the $\mathrm{NC}$ and overexpressed miR-124 groups were significantly increased in 

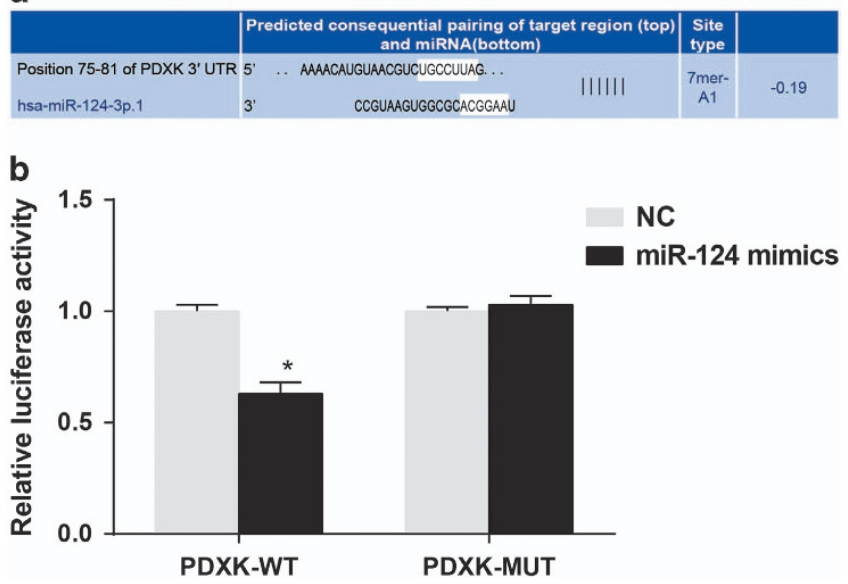

Figure 4 The identification of PDXK as the target gene of miR-124 using a luciferase reporter gene assay. (a) The identification of PDXK as the target gene of miR-124 using the online software Targetscan 7.1. (b) A comparison of the luciferase activity among cells transfected with PDXK-WT/PDXK-MUT+NC oligonucleotide or PDXK-WT/PDXK-MUT+miR-124 mimics. miR-124, microRNA-124; MUT, mutant type; NC, negative control; WT, wild type. ${ }^{*} P<0.05$ compared with cells transfected with $P D X K-\mathrm{WT}+\mathrm{NC}$ oligonucleotide.

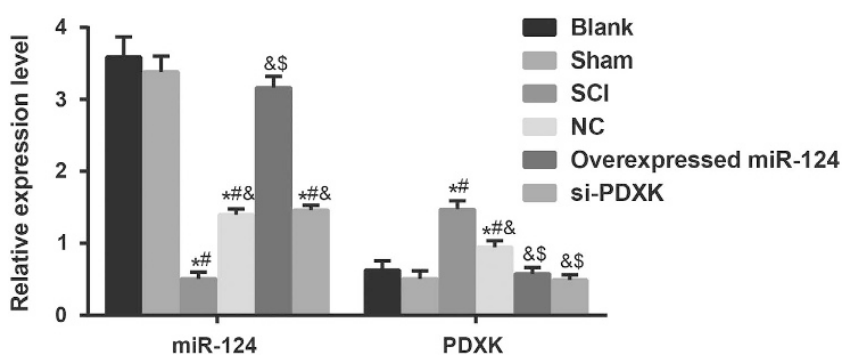

Figure 5 Comparisons of the mRNA expression of miR-124 and PDXK among the blank, sham, SCI, NC, overexpressed miR-124 and si-PDXK groups. miR-124, microRNA-124; NC, negative control; SCl, spinal cord injury; si-PDXK, PDXK-siRNAs. ${ }^{*} P<0.05$ compared with the blank group; ${ }^{\#} P<0.05$ compared with the sham group; \& $P<0.05$ compared with the $\mathrm{SCl}$ group $P<0.05 ;{ }^{\$} P<0.05$ compared with the NC group.

comparison to that of the SCI group (all $P<0.05$ ), and the overexpressed miR-124 group had a remarkably higher BBB score than the NC group $(P<0.05)$. Moreover, 35 days after transplantation, the BBB scores in the SCI and NC groups were significantly reduced compared to those of the blank and sham groups (all $P<0.05$ ), and no significant differences were observed among the BBB scores of the overexpressed miR-124, the blank and sham groups (all $P>0.05$ ). The experimental results above showed that miR-124 overexpression or PDXK gene silencing improved the ability of BMSCs to repair SCI.

Comparison of nerve cell markers and BrdU-positive cells among the blank, sham, SCI, NC, overexpressed miR-124 and si-PDXK groups

On 35th day after SCI, the results showed that no BrdUpositive cells were observed in the blank, sham and SCI groups, but BrdU-positive cells were observed in the overexpressed miR-124, NC and si-PDXK groups. In addition, the doublelabeled positive cell density (BrdU+NSE, BrdU+NF and BrdU +MAP2), the BrdU-positive cell density and the ratio of BrdUpositive cells/nerve cell marker-positive cells to BrdU-positive cells/nerve cell marker-negative cells in the overexpressed miR-124 group were significantly higher than those of the NC group (all $P<0.05$ ) (Figure 7). Thus, miR-124 overexpression or PDXK gene silencing may enhance the differentiation of BMSCs into neurocytes.

Comparisons of protein expression levels of SCI repairrelated proteins among the blank, sham, SCI, NC, overexpressed miR-124 and si-PDXK groups

The protein expression levels of TRH, PGI2 and GM in the NC group were significantly higher than those in the SCI group (all $P<0.05$ ), and the protein expression levels of TRH, PGI2 and GM in the overexpressed miR-124 group were significantly increased compared with the NC group (all $P<0.05$ ) (Figure 8). These results indicated that miR-124 overexpression or PDXK gene silencing increased the expression of SCI repair-related proteins, including TRH, PGI2 and GM.

\section{DISCUSSION}

The mechanism of miR-124-modified BMSC transplantation in the repair of SCI in rats was investigated in a rat model of SCI. This study found that miR-124 accelerated the differentiation of BMSCs into neurocytes and promoted the repair of SCI. Thus, miR-124 is expected to become a new target for SCI treatment.

Our study found that there was a significant decrease in the miR-124 expression in the SCI group. miR-124 is expressed in both the immature and mature neurons of the nervous system, and is relatively abundant in the brain, retina and spinal cord neurons; it is also critical in neural development and differentiation. ${ }^{17} \mathrm{~A}$ decrease in the level of miR-124 was observed in the brain and spinal cord tissues of mice after $\mathrm{SCI}$, and its expression may reflect the severity of SCI. ${ }^{18}$ A study by Nakanishi et al. ${ }^{19}$ discovered that miR-124a was specifically expressed in the central nervous system, but there was a significant reduction in the miR-124a expression in rats with SCI, which was consistent with this study. In addition, the protein expression levels of PDXK in the overexpressed miR-124 and si-PDXK groups were significantly lower when compared with the SCI and NC groups, indicating that the overexpression of miR-124 inhibited the expression of PDXK. PDXK is capable of catalyzing the phosphorylation of vitamin B6, resulting in its active form, pyridoxal phosphate; it participates in the salvage synthesis pathway of pyridoxal phosphate in order to maintain the normal physiological function of the central nervous system. Metabolic disorder of PDXK activity can result in metabolic disorder of amino acids, which may be the pathogenesis of the secondary injury of nervous system and other neurological damage. ${ }^{20,21}$ PDXK is distributed in the cytoplasm of spinal cord neurons and glial cells, which maintain the dynamic equilibrium of the pyridoxal 
phosphate concentration to restore stability of the SCI microenvironment, and PDXK is also the target protein for miR-124 to inhibit. ${ }^{22}$ Therefore, it was found that miR-124 was a negative regulator of PDXK, and it conferred an inhibitory effect on PDXK expression. Consistent with this finding, the luciferase activity of cells treated with $\mathrm{NC}+P D X K-\mathrm{WT}$ was significantly higher than that of the cells treated with miR-124 mimics $+P D X K-W T$. In addition, the Targetscan software
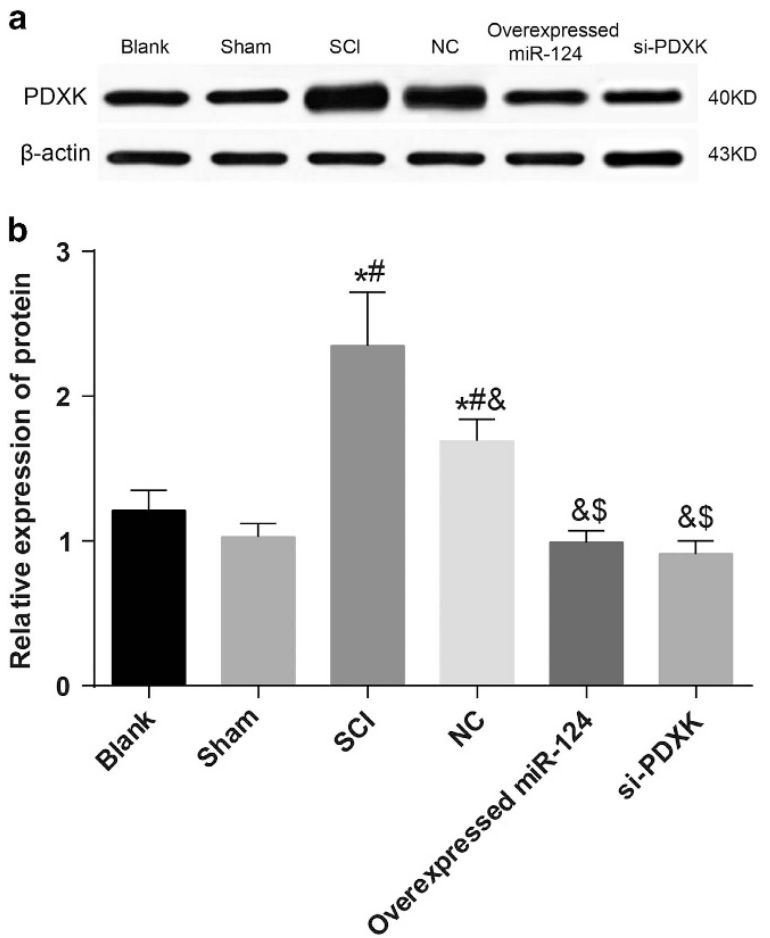

Figure 6 A comparison of the PDXK protein expression among the blank, sham, SCl, NC, overexpressed miR-124 and si-PDXK groups. (a) The band analysis of the PDXK protein expression among the blank, sham, SCI, NC, overexpressed miR-124 and si-PDXK groups detected by western blotting. (b) A comparison of the PDXK protein expression among the blank, sham, $\mathrm{SCl}, \mathrm{NC}$, overexpressed miR-124 and si-PDXK groups. miR-124, microRNA-124; NC, negative control; PDXK, pyridoxal kinase; SCI, spinal cord injury; si$P D X K, \quad P D X K$-siRNAs. ${ }^{*} P<0.05$ compared with the blank group; $\# P<0.05$ compared with the sham group; \& $P<0.05$ compared with the $\mathrm{SCl}$ group; $\$ P<0.05$ compared with the NC group. indicated that PDXK was the target gene of miR-124. However, the specific mechanism of the regulation of PDXK by miR-124 requires further research.

The current study also found that the BBB scores were significantly increased in the overexpressed miR-124 and si-PDXK groups compared with the SCI group at 7, 21 and 35 days after transplantation. The BBB scale has been widely used to evaluate the functional recovery of rats after SCI. ${ }^{23}$ In the overexpressed miR-124 and si-PDXK groups, a few necrotic neurons in the spinal cord gray matter, the complete axonal structure in the white matter axonal and the clear lamellar structure in the myelin were observed. These results confirmed that miR-124 overexpression or PDXK gene silencing improved the ability of BMSCs to repair injured spinal cord tissues.

In addition, the double-labeled positive cell density (BrdU+NSE, BrdU+NF or BrdU+MAP2), the BrdU-positive cell density and the ratio of BrdU-positive cells/nerve cell marker-positive cells to the BrdU-positive cells/nerve cell marker-negative cells in the overexpressed miR-124 group were significantly higher than those in the NC group. NSE, $\mathrm{NF}$ and MAP2 are neuronal markers involved in neuronal differentiation, and they have been used to assess the differentiation of BMSC into neuronal cells. ${ }^{24,25}$ Thus, miR-124 overexpression could enhance the differentiation of BMSCs into neurons. The miR-124 in neural stem cells (NSCs) could promote NSC differentiation into neurons and astrocytes, which could fill the cleaved neurons and astrocytes in the damaged area. NSCs could also secrete a variety of neurological nutritional factors needed for SCI repair, which is conducive to improving the environment and the restoration of functional recovery after SCI. ${ }^{11}$ Thus, miR-124 might improve the differentiation competence of BMSCs into neurocytes, which thereby could enhance the capability of BMSCs to repair SCI. NSCs could construct new synaptic connections by connecting SCI ends, secrete neurotrophic factors in the injured area, improve the damaged spinal microenvironment, and promote the remyelination and recovery of nerve conduction to repair SCI. ${ }^{26}$ miRNAs could mediate the neural differentiation of stem cells, and the brain-specific miR miR-124 is considered to be a mediator of neurogenesis. ${ }^{27}$ Importantly, miR-124 may be associated with the differentiation of BMSCs in that the

Table 2 Comparison of BBB scores at different time points among the blank, sham, SCI, NC, overexpressed miR-124 and si-PDXK groups

\begin{tabular}{|c|c|c|c|c|c|c|}
\hline Time point & Blank group & Sham group & SCl group & NC group & $\begin{array}{l}\text { Overexpressed } \\
\text { miR-124 group }\end{array}$ & si-PDXK group \\
\hline 24 Hours after being injured & $16.75 \pm 1.98$ & $15.98 \pm 2.17$ & $2.86 \pm 0.95^{a, b}$ & $3.15 \pm 1.01^{\mathrm{a}, \mathrm{b}}$ & $2.53 \pm 0.56^{a, b}$ & $2.69 \pm 0.62^{a, b}$ \\
\hline 21 Days after inoculation & $18.11 \pm 2.36$ & $16.52 \pm 2.15$ & $4.83 \pm 1.12^{\mathrm{a}, \mathrm{b}}$ & $9.02 \pm 1.75^{a, b, c}$ & $12.99 \pm 2.51^{\mathrm{a}, \mathrm{b}, \mathrm{c}, \mathrm{d}}$ & $13.41 \pm 2.87^{a, b, c, d}$ \\
\hline 35 Days after inoculation & $18.34 \pm 2.43$ & $18.27 \pm 2.61$ & $6.95 \pm 1.08^{a, b}$ & $12.37 \pm 1.31^{a, b, c}$ & $17.55 \pm 2.42^{c, d}$ & $17.94 \pm 2.36^{c, d}$ \\
\hline
\end{tabular}

Abbreviations: BBB, Basso, Beattie and Bresnahan; miR-124, microRNA-124; NC, negative control; SCI, spinal cord injury; si-PDXK, PDXK-siRNA.

a $P<0.05$ compared with the blank group at the same time point.

${ }^{\mathrm{b}} P<0.05$ compared with the sham group at the same time point.

${ }^{c} P<0.05$ compared with the SCl group at the same time point.

$\mathrm{d} P<0.05$ compared with the NC group at the same time point. 
a
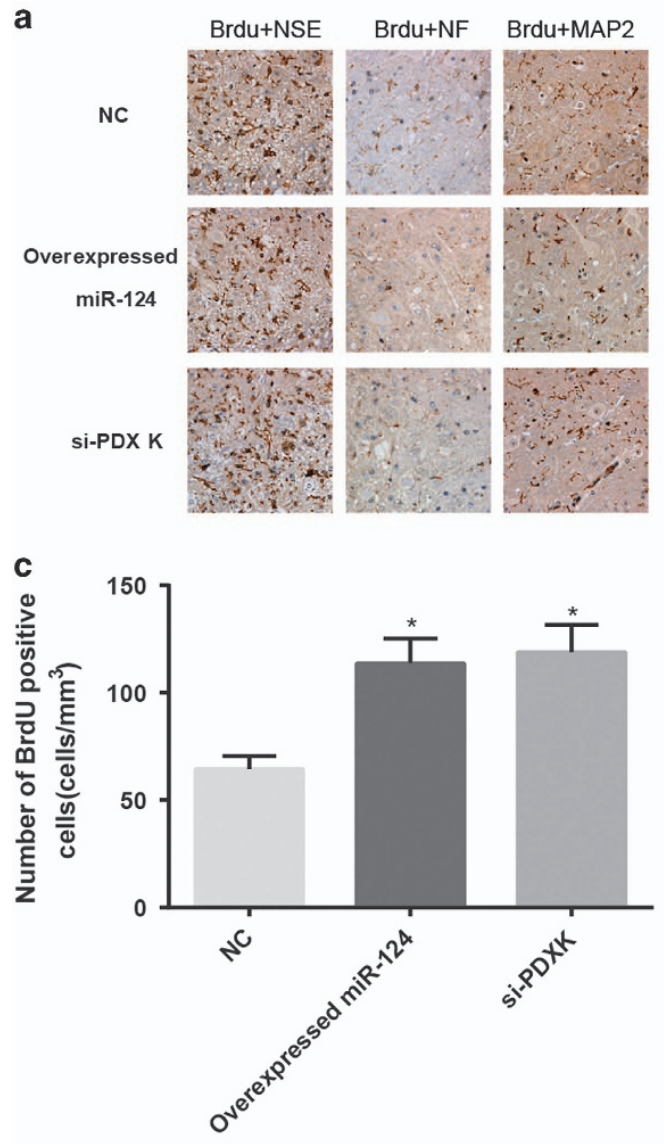

b

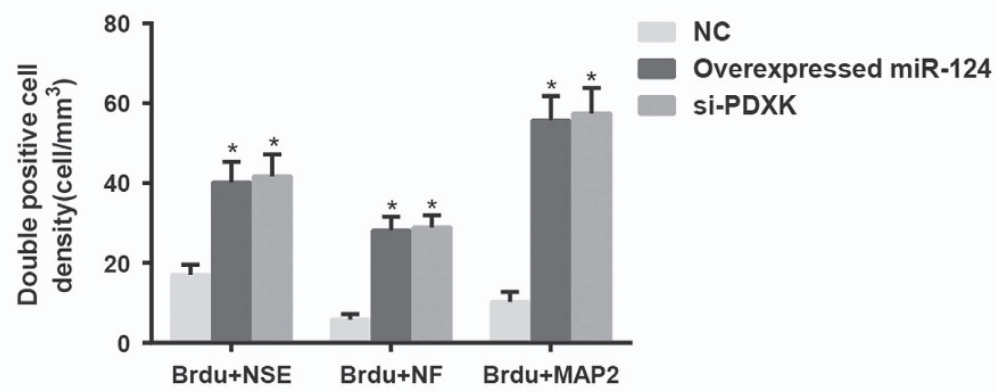

d

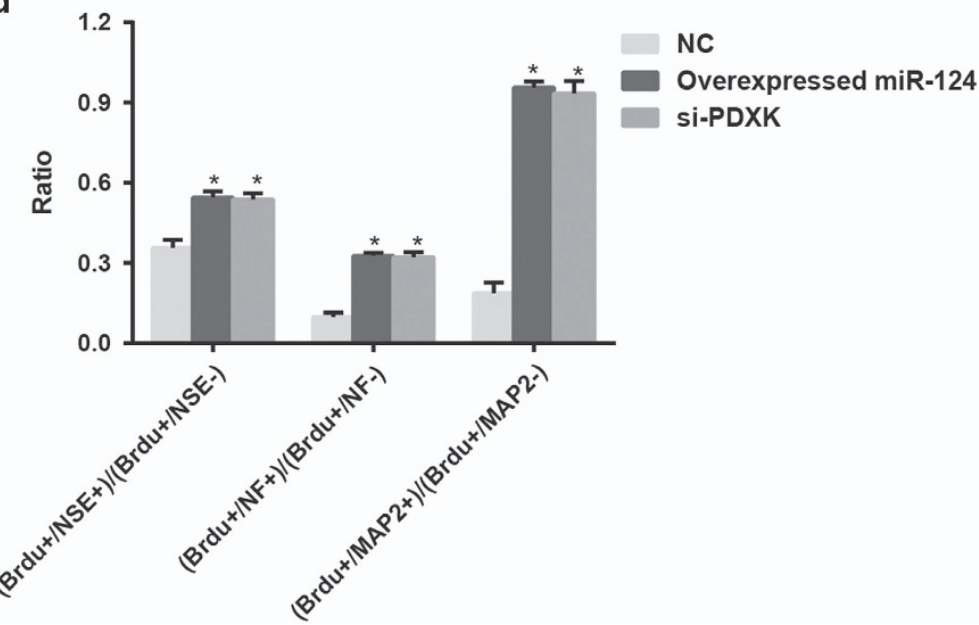

Figure 7 Comparisons of the nerve cell markers and BrdU-positive cells among the NC, overexpressed miR-124 and si-PDXK groups. (a) Double-labeled positive cells (BrdU+NSE, BrdU+NF and BrdU+MAP2) detected by immunohistochemistry among the NC, overexpressed miR-124 and si-PDXK groups. (b) A comparison of the double-labeled positive cell density (BrdU+NSE, BrdU+NF and BrdU +MAP2) among the NC, overexpressed miR-124 and si-PDXK groups. (c) A comparison of the BrdU-positive cell density among the NC, overexpressed miR-124 and si-PDXK groups. (d) A comparison of the ratio of BrdU-positive cells/nerve cell marker-positive cells to the BrdU-positive cells/nerve cell marker-negative cells among the NC, overexpressed miR-124 and si-PDXK groups. BrdU, bromodeoxyuridine; MAP2, microtubule-associated protein 2; miR-124, microRNA-124; NC, negative control; NF, neurofilament; NSE, neuron-specific enolase; si-PDXK, PDXK-siRNAs. ${ }^{*} P<0.05$ compared with the NC group.

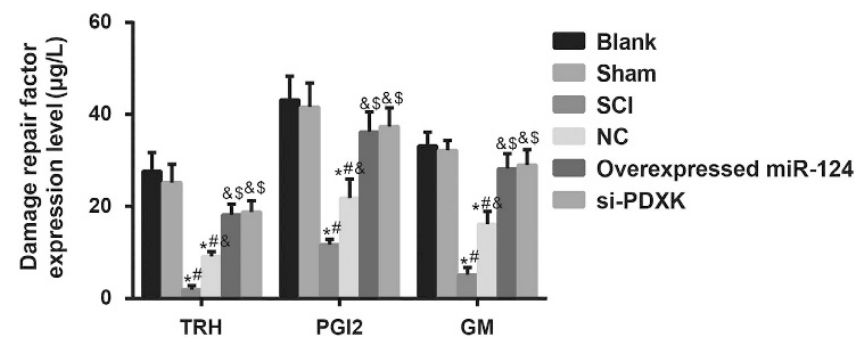

Figure 8 Comparisons of the protein expression levels of $\mathrm{SCl}$ repairrelated proteins among the blank, sham, $\mathrm{SCl}, \mathrm{NC}$, overexpressed miR-124 and si-PDXK groups. miR-124, microRNA-124; NC, negative control; SCl, spinal cord injury; si-PDXK, PDXK-siRNAs. ${ }^{*} P<0.05$ compared with the blank group; ${ }^{\#} P<0.05$ compared with the sham group; \& $P<0.05$ compared with the $\mathrm{SCl}$ group; $\$ P<0.05$ compared with the NC group.

overexpression of miR-124 promotes the differentiation of BMSCs into neurons. ${ }^{28}$ In addition, the inhibition of miR-124 would not only reduce the neuronal differentiation efficiency of
BMSCs but also reduce cell viability. ${ }^{29}$ For further comprehension of this role, our study also detected the protein expression levels of SCI repair-related proteins (TRH, PGI2 and GM). The expression levels of these proteins in the overexpressed miR-124 group were significantly higher than those of the NC and SCI groups. Several studies have proposed that the association of TRH, PGI2 and GM with SCI could contribute to the possible treatments. ${ }^{30-32}$ However, the explanation of the detailed mechanism for this association is limited, and more studies are required in the future.

In conclusion, this study demonstrates that miR-124 accelerates the differentiation ability of BMSCs into neurocytes, thereby promoting the repair of spinal cord tissues after SCI. This finding may provide a new target for SCI therapy. The overexpression of miR-124 inhibited the expression of PDXK, which could be a possible mechanism for how miR-124 promotes the differentiation of BMSCs. However, further research of this mechanism is required, and the 
role of miR-124 downstream signaling pathways requires further study.

\section{CONFLICT OF INTEREST}

The authors declare no conflict of interest.

\section{ACKNOWLEDGEMENTS}

We thank all the people involved in editing the paper.

1 Kirshblum SC, Burns S, Biering-Sorensen F, Donovan W, Graves DE, Jha A et al. International standards for neurological classification of spinal cord injury, revised 2011. Top Spinal Cord Inj Rehabil 2012; 18: 85-99.

2 Katoh S, Enishi T, Sato N, Sairyo K. High incidence of acute traumatic spinal cord injury in a rural population in Japan in 2011 and 2012: an epidemiological study. Spinal Cord 2014; 52: 264-267.

3 Salazar DL, Uchida N, Hamers FP, Cummings BJ, Anderson AJ. Human neural stem cells differentiate and promote locomotor recovery in an early chronic spinal cord injury NOD-SCID mouse model. PLOS ONE 2010; 5: e12272.

4 Spinal Cord Injury (SCI) 2016 facts and figures at a glance. J Spinal Cord Med 2016; 39: 493-494.

5 Shichinohe H, Kuroda S, Tsuji S, Yamaguchi S, Yano S, Lee JB et al. Bone marrow stromal cells promote neurite extension in organotypic spinal cord slice: significance for cell transplantation therapy. Neurorehabil Neural Repair 2008; 22: 447-457.

6 Tosolini AP, Morris R. Viral-mediated gene therapy for spinal cord injury (SCl) from a translational neuroanatomical perspective. Neural Regen Res 2016; 11: 743-744.

7 Gao Y, Peng J, Ren Z, He NY, Li Q, Zhao XS et al. Functional regulatory roles of microRNAs in atherosclerosis. Clin Chim Acta 2016; 460: 164-171.

8 Furuta M, Kozaki KI, Tanaka S, Arii S, Imoto I, Inazawa J. miR-124 and miR-203 are epigenetically silenced tumor-suppressive microRNAs in hepatocellular carcinoma. Carcinogenesis 2010; 31: 766-776.

9 Gao XR, Wang HP, Zhang SL, Wang MX, Zhu ZS. Pri-miR-124 rs531564 polymorphism and colorectal cancer risk. Sci Rep 2015; 5: 14818.

10 Cheng LC, Pastrana E, Tavazoie M, Doetsch F. miR-124 regulates adult neurogenesis in the subventricular zone stem cell niche. Nat Neurosci 2009; 12: 399-408.

11 Xu W, Li P, Qin K, Wang X, Jiang X. miR-124 regulates neural stem cells in the treatment of spinal cord injury. Neurosci Lett 2012; 529: 12-17.

12 Czarnecka J, Porowinska D, Bajek A, Holysz M, Roszek K. Neurogenic differentiation of mesenchymal stem cells induces alterations in extracellular nucleotides metabolism. J Cell Biochem 2016; 118: 478-486.

13 Zhou LL, Liang JK, Lin QM, Huang ZT. Effect and mechanism of different ways of transplanting bone marrow mesenchymal stem cells in cardiopulmonary resuscitation in rats. Genet Mol Res 2014; 13: 7937-7949.

14 Wang W, Li B, Yang J, Xin L, Li Y, Yin $\mathrm{H}$ et al. The restoration of full-thickness cartilage defects with BMSCs and TGF-beta 1 loaded PLGA/fibrin gel constructs. Biomaterials 2010; 31: 8964-8973.

15 Li F, Fei D, Sun L, Zhang S, Yuan Y, Zhang L et al. Neuroprotective effect of bone marrow stromal cell combination with atorvastatin in rat model of spinal cord injury. Int J Clin Exp Med 2014; 7: 4967-4974.

16 Basso DM, Beattie MS, Bresnahan JC. A sensitive and reliable locomotor rating scale for open field testing in rats. $J$ Neurotrauma $1995 ; 12: 1-21$.

17 Yu JY, Chung KH, Deo M, Thompson RC, Turner DL. MicroRNA miR-124 regulates neurite outgrowth during neuronal differentiation. Exp Cell Res 2008; 314: 2618-2633.
18 Zhao Y, Zhang $\mathrm{H}$, Zhang $\mathrm{D}$, Yu CY, Zhao XH, Liu FF et al. Loss of microRNA-124 expression in neurons in the peri-lesion area in mice with spinal cord injury. Neural Regen Res 2015; 10: 1147-1152.

19 Nakanishi K, Nakasa T, Tanaka N, Ishikawa M, Yamada K, Yamasaki K et al. Responses of microRNAs 124a and 223 following spinal cord injury in mice. Spinal Cord 2010; 48: 192-196.

20 Safo MK, Musayev FN, di Salvo ML, Hunt S, Claude JB, Schirch V. Crystal structure of pyridoxal kinase from the Escherichia coli pdxK gene: implications for the classification of pyridoxal kinases. J Bacteriol 2006; 188: 4542-4552.

21 Moreno-Navarrete JM, Jove M, Ortega F, Xifra G, Ricart W, Obis E et al. Metabolomics uncovers the role of adipose tissue PDXK in adipogenesis and systemic insulin sensitivity. Diabetologia 2016; 59: 822-832.

22 Mittenhuber G. Phylogenetic analyses and comparative genomics of vitamin B6 (pyridoxine) and pyridoxal phosphate biosynthesis pathways. J Mol Microbiol Biotechnol 2001; 3: 1-20.

23 Yamaya S, Ozawa H, Kanno H, Kishimoto KN, Sekiguchi A, Tateda S et al. Low-energy extracorporeal shock wave therapy promotes vascular endothelial growth factor expression and improves locomotor recovery after spinal cord injury. J Neurosurg 2014; 121: 1514-1525.

24 Yan T, Skaftnesmo KO, Leiss L, Sleire L, Wang J, Li X et al. Neuronal markers are expressed in human gliomas and NSE knockdown sensitizes glioblastoma cells to radiotherapy and temozolomide. BMC Cancer 2011; 11: 524 .

25 Sun Z, Li X, Su Z, Zhao Y, Zhang L, Wu M. Electroacupuncture-enhanced differentiation of bone marrow stromal cells into neuronal cells. J Sport Rehabil 2009; 18: 398-406.

26 Martino G, Pluchino S. The therapeutic potential of neural stem cells. Nat Rev Neurosci 2006; 7: 395-406.

27 Krichevsky AM, Sonntag KC, Isacson 0, Kosik KS. Specific microRNAs modulate embryonic stem cell-derived neurogenesis. Stem Cells 2006; 24: 857-864.

28 Zou D, Chen Y, Han Y, Lv C, Tu G. Overexpression of microRNA-124 promotes the neuronal differentiation of bone marrow-derived mesenchymal stem cells. Neural Regen Res 2014; 9: 1241-1248.

29 Santos MC, Tegge AN, Correa BR, Mahesula S, Kohnke LQ, Qiao M et al. miR-124, -128 , and -137 orchestrate neural differentiation by acting on overlapping gene sets containing a highly connected transcription factor network. Stem Cells 2016; 34: 220-232.

30 Baykal S, Ceylan S, Usul H, Akturk F, Deger O. Effect of thyrotropinreleasing hormone on $\mathrm{Na}(+)-\mathrm{K}(+)$-adenosine triphosphatase activity following experimental spinal cord trauma. Neurol Med Chir 1996; 36: 296-299.

31 Harada N, Taoka Y, Okajima K. Role of prostacyclin in the development of compression trauma-induced spinal cord injury in rats. J Neurotrauma 2006; 23: 1739-1749.

32 Xu D, Yang L, Li Y, Sun Y. Clinical study of ganglioside (GM) combined with methylprednisolone (MP) for early acute spinal injury. Pak J Pharm Sci 2015; 28: 701-704.

$($ (1) $\circledast)$ This work is licensed under a Creative Commons Attribution-NonCommercial-NoDerivs 4.0 International License. The images or other third party material in this article are included in the article's Creative Commons license, unless indicated otherwise in the credit line; if the material is not included under the Creative Commons license, users will need to obtain permission from the license holder to reproduce the material. To view a copy of this license, visit http://creativecommons.org/licenses/by-nc-nd/4.0/ 